\title{
M orbidade neonatal e maternas relacionada ao tipo de parto
}

\author{
Neonatal and maternal morbidity related to the type of delivery
}

Priscila O liveira Cardoso $^{1}$

Luiz Ronaldo Alberti ${ }^{2}$

Andy Petroianu ${ }^{2}$

\footnotetext{
${ }^{1}$ Departamento deCirurgia, UniversidadeFederal de Minas Gerais. Av. Professor Alfredo Balena 190/sala 203, Santa Efigênia. 30130-100 Belo Horizonte, M G ocpriscila@gmail.com 2Departamento de Cirurgia, UniversidadeFederal de M inas Gerais.
}

Abstract An evaluation of infant morbimortality and mother morbidity was undertaken according to the type of delivery. A prospective study was undertaken on 170 puerperal patients divided into two groups: Group $1(n=95)$, natural or vaginal delivery, Group $2(n=75)$, submitted to caesarean delivery. Complications in mothers were classified in small, moderate and severe. The infant parameters were: period of pregnancy, weight on birth, A pgar score, necessity of intensive care and neurological disorders. M others who had not completed elementary school $(p=0.0045)$ had more vaginal delivery. Previous vaginal deliveries were more common in Group 1 than caesarean section in Group 2 $(p=<0.001)(O R=104.00 ; 21.11<0 R<$ 610.99). In Group 1, vaginal delivery waspreferred by $78(82,1 \%)$ of mothers comparingto $28(37.3 \%)$ from Group 2, who preferred caesarean section ( $p$ $=0.0002)(O R=4 ; 1.77<O R<9.17)$. Post-operative was more intense and frequent after caesare an section ( 8 cases) than patients of Group 1 (2 cases) $(p=0.018)(O R=0.18 ; 0.03<0 R<0.96)$. Obstetric trauma was found in 14 deliveries of Group 1 and 7 of Group $2(p=0.28)$. Infant hospitalization was greater in Group $2(3.43+/-0.70$ days) in comparison with Group 1 ( $2.71+$ +/- 0.67 days) $(p<0.0001)$. Theinfant morbidity was greater after vaginal deliveries, but maternal morbidity was greater after caesarean deliveries.

Key words Infant, Parturient, M orbidity, Vaginal delivery, Caesarean delivery
Resumo Foi realizada uma análise da morbidade neonatal e materna e a mortalidade neonatal de acordo com o tipo de parto, cesariana ou vaginal. Foram estudadas prospectivamente 170 parturientes sem complicações gestacionais e com nascimento a termo: Grupo $1(n=95)$, puérpera com parto por via vaginal, Grupo $2(n=75)$, puérpera submetida à cesariana. Parâmetros maternos e fetais foram avaliados. Foi observada maior incidência de partos por via vaginal nas pacientes que estudaram até 0 ensino fundamental incompleto $(p=0,0045)$. H ouve prevalência maior de partos prévios por via vaginal no Grupo 1 e de cesáreas no Grupo 2 ( $p<0,001)$. O bservou preferência de $78(82,1 \%)$ das mulheres do Grupo 1 pelo parto vaginal contra apenas $28(37,3 \%)$ dasmulheresdo Grupo 2, pela cesárea $(p=0,0002)$. Houve dor intensa no pós-operatório nas pacientes submetidas a parto cesáreo (oito casos) ( $p=$ 0,018 ). Traumas obstétricos foram verificados em catorze recém-nascidos do Grupo 1 e em sete do Grupo $2(p=0,28)$. Concluímosqueexistemaior morbidade neonatal em recém-natos de parto por via vaginal quando comparada com neonatos de cesárea e maior morbidade materna em puérpe ras com parto cesariana.

Palavras-chave Neonato, Parturiente, M orbimortalidade neonatal, Parto normal, Cesariana 
Introdução

A té cerca de trinta anos atrás, a maioria dos partos ocorria por via vaginal, conhecido como "parto normal". Havia menos médicos adeptos à cesariana e sua execução envolvia riscos anestésicos e cirúrgicos, agravados pelo caráter de urgência das indicações desse tipo de parto ${ }^{1}$.

$N$ as últimas décadas, houve importantes avanços na assistência médica a parturientes, possibilitando maior segurança para as mães e os fetos. Entretanto, essa situação favorável acompanhou-se de grande aumento nas indicações de cesáreas em todo o mundo. 0 Canadá apresentou 21,2\% de partos por cesáreas em 2001, enquanto nos Estados Unidos, o percentual de cesarianas atingiu 26,1\% em 20022,3.

Em estudo realizado por Belizán et al. ${ }^{4}$ na dé cada de noventa, verificou-se que o Brasil ocupava o segundo lugar entre os doze países da América Latina com maior incidência decesarianas. Atualmente, o Brasil apresenta uma taxa global de cesarianas de $32 \%$, chegando a $90 \%$ em algumas clínicas privadas ${ }^{2,5}$. No Estado de São Paulo, a taxa de cesarianas alcançou $32,9 \%$ no setor público e $80,4 \%$ no setor privado 6 . A diminuição na mortalidade neonatal não correspondeu ao aumento nas taxas decesáreas, o que contraria a justificação da prática indiscriminada desse procedimento, como sendo mais seguro. Acredita-se que os principaisfatores determinantes da queda da morbimortalidade neonatal te nham sido o desenvolvimento das técnicas de terapia intensiva neonatal e a melhoria da atenção à saúde materna, que não mantêm relação direta com a via de parto?

Há, na literatura, a descrição de vários fatores que influenciam na incidência de cesarianas, como a remuneração do parto, aspectos culturais, falta de tempo dos obstetras ea forma como está estruturada a assistência hospitalar de saúde pública e a conveniada 8 . Constatou-se que 0 parto normal foi realizado cerca de quatro vezes mais em hospitais públicos, em relação aos hospitais privados sem atendimento pelo Sistema Ú nico de Saúde (SUS) e $24 \%$ a mais nos hospitais públicos em relação aos hospitais privados com atendimento pelo SU S9. Esses dados indicam que há relação entre a situação socioeconômica e a escolha do tipo de parto ${ }^{10}$.

A tendência dos obstetras tem sido preferir parto por cesariana, tanto a pedido da gestante quanto por vantagens para eles próprios. A demanda da paciente por uma cesariana é sustentada no medo, na conveniência e na desinforma- ção $0^{2,11}$. Muitas vezes, a gestante receia as consequências do parto por via vaginal, por considerá-lo uma experiência arriscada. A mulher tem a idéia paradoxal dequeo ato cirúrgico éum modo para evitar a dor ${ }^{2}$. Para o obstetra, o parto agendado é mais conveniente uma vez que, no parto normal, o médico tem que abdicar das outras tarefas para dedicar várias horas ao atendimento da parturiente ${ }^{9,12}$. Além disso, houve um aumento na segurança desse ato cirúrgico ${ }^{1}$.

Em estudo realizado por M c Farland et al. ${ }^{13}, 0$ parto por cesárea foi associado a efeito protetor para o neonato, por diminuição do risco de lesão do plexo braquial e de fraturas ósseas ${ }^{14}$. Em relação à parturiente, o risco de incontinência urinária é menor e as lesões perineais são prevenidas nas mulheres que se submeteram a cesarianas ${ }^{15}$.

Contudo, ainda existem muitos médicos que defendem o parto por via vaginal como primeira escolha, por considerarem ter ele muitas vantagens em relação à cesariana, principalmente por ser fisiológico, tendo em vista que o corpo da mulher foi preparado para tal. A recuperação após o parto natural émais rápida e há menor chance de hematomas, infecções ou outras complicações cirúrgi cas para a mãe. A maioria das parturientes e dos neonatos é capaz de atravessar de maneira saudável o crítico momento do nascimento, sem necessidade de intervenção médica ${ }^{1}$. Quanto à dor, as técnicas de anal gesia atuais previnem esse desconforto.

Os dados de literatura não parecem consistentes para justificar que uma cesariana sem indicação médica deva ser feita como profilaxia para dano no neonato ${ }^{1,5}$. Segundo Rouse et al. ${ }^{16}$, seriam necessárias centenas de partos por cesárea para evitar-se um único dano de plexo braquial permanente. Além disso, neonatos a termo nascidos por cesariana eletiva têm risco aumentado de desenvolver desordens respiratórias comparadas com aqueles nascidos por parto normal ${ }^{17,18}$. Com rel ação à amamentação, observou-se que os nascidos por cesárea eletiva apresentam uma chance três vezes maior de interrupção completa da lactação aos trinta dias de vida' ${ }^{19}$. A morbidade da parturiente pós-parto cesariana são maiores que para nascimentos por via vaginal. Comparado ao parto por via vaginal espontâneo, a Cesário está associada a riscos aumentados de infecções, necessidade de transfusão sanguínea, pneumonia, complicações cardiopulmonares, tromboembolismo e desordens gastrointestinais na parturiente ${ }^{3,5,20-22}$. 0 risco de readmissão hospitalar nos sessenta dias pós parto cesarianaémaior queapós o parto por via vaginal espontânea. 
Alguns autores consideram um aumento exagerado no número de partos Cesário, com cerca de oitocentos mil desnecessários a cada ano na América Latina ${ }^{4}$.

0 objetivo do presente trabalho foi analisar prospectivamente a morbidade e a mortalidade neonatais de acordo com o tipo de parto, cesariana ou por via vaginal (considerando também o uso de fórceps), em diferentes idades gestacionais. Foram verificadas também complicações maternas decorrentes do parto.

\section{Método}

Este trabalho foi realizado de acordo com as recomendações da Declaração de H elsinque e da Resolução n 196/96 do M inistério da Saúde sobre pesquisa envolvendo seres humanos e foi aprovada pelo Comitê de Ética em Pesquisa da Universidade Federal de Minas Gerais (UFM G) (23). Todos os pacientes concordaram em participar do estudo, por meio de consentimento livre eesclarecido.

Foram estudadas prospectivamente 170 pacientes no período puerperal, na M aternidade Municipal de Contagem e na M aternidade da Fundação H ospitalar N ossa Senhora de Lourdes em N ova Lima, entre janeiro de 2006 a dezembro de 2006. 0 estudo foi conduzido por meio de entrevistas, após consentimento informado. A veracidade dos dados foi estimulada pela garantia de sigilo quanto à identificação dos entrevistados.

Foram avaliadas parturientes sem complicações gestacionais ecom nascimento a termo, distribuídas em dois grupos de acordo com o tipo de parto:

Grupo $1(n=95)$ : puérperas com parto por via vaginal

Grupo $2(n=75)$ : puérperas submetidas a cesáreas.

Cada mulher foi entrevistada no período puerperal. Foi utilizado o mesmo questionário para os dois grupos. Além da entrevista, houve coleta de dados dos prontuários da parturientee do recém-nato. Foram excluídos todos os casos em que se verificou doença do feto, seu baixo peso corpóreo, traumas gestacionais ou ainda afecções maternas que poderiam repercutir no feto ou no recém-nascido.

No estudo das condições maternas, registraram-se a idade, a religião, o estado civil, a atividade profissional, o grau de instrução, história gestacional, doenças pregressas, tratamentos médicos, operações pregressas, alergias, tabagismo e etilismo.
$\mathrm{Na}$ avaliação das condições gestacionais, verificou-se história pregressa gestacional, tipos de partos prévios, número de consultas pré-natais, tipo de parto desejado no pré-natal e a justificação da escolha pela parturiente e pelo médico.

$\mathrm{Na}$ análise da via de parto, foram consideradas a idade gestacional, peso ao nascer, duração da internação na unidade neonatal, categoria do hospital, consultas pré-natais enota de Apgar, que mostra as condições vitais do neonato. Considerou-se como baixo peso ao nascer os recémnatos com peso inferior a $2.500 \mathrm{~g}$; como período neonatal, os primeiros 28 dias devida e como pré termo, a idade gestacional ao nascer inferior a 36 semanas. Verificaram-se a presença de afecção respiratória neonatal, a necessidade de cuidado intensivo e desordens neurológicas. Levou-se em consideração o tipo de anestesia e a duração de fase de expulsão, prolapso do cordão umbilical.

A avaliação estatística utilizou o método descritivo de média e desvio padrão da média para a idade dos pacientes. A comparabilidade dos gruposteve por basea idadedas pacientes. As pacientes estudadas foram comparadas pelos testes de normalidade de Kolmogorov-Smirnov, de t de Student para amostras contínuas não pareadas, qui-quadrado eexato deFisher. Calculou-setambém 0 odds ratio e 0 intervalo de confiança das variáveis analisadas. As diferenças foram consideradas significativas para val ores correspondentes a $p<0,05$.

\section{Resultados}

A idade das pacientes apresentou valores com distribuição dentro da curva normal $(p>0,10)$ (distância KS de 0,1054 para o Grupo 1, KS de 0,1161 para o Grupo 2). Ela variou entre 16 e 43 $(24,83 \pm 6,03)$ anos no Grupo 1 e entre 14 e 38 $(26,65 \pm 6,84)$ anos, no Grupo 2 , sem diferença entresi $(p=0,0771)$.

A Tabela 1 mostra parâmetros relacionados à mãe avaliados nos dois grupos de pacientes estudados. O bserva-se que não houve diferença entre os grupos em relação à crença religiosa ( $p$ $=0,1458$ ). Foi verificada uma incidência maior de católicos seguidos pelos evangélicos em ambos os grupos.

União estável foi relatada em 72 (75,7\%) pacientes do Grupo 1 e em 55 (73,3\%) pacientes do Grupo 2. 0 estado civil não interferiu no tipo de parto $(p=0,7145)(O R=0,88 ; 0,41<O R<1,86)$. A maioria $(55,7 \%)$ das pacientes do Grupo $1 \mathrm{e}$ 48\% das pacientes do Grupo 2 desempenhavam 


\begin{tabular}{|c|c|c|c|}
\hline \multicolumn{4}{|c|}{$\begin{array}{l}\text { Tabela 1. Dados epidemiológicos das pacientes submetidas a } \\
\text { partos por via vaginal (Grupo 1) e por cesárea (Grupo } 2 \text { ). }\end{array}$} \\
\hline & Grupo 1 & Grupo 2 & Valor $p$ \\
\hline Religião & & & 0,145 \\
\hline Católicos & 53 & 35 & \\
\hline Evangélicos & 34 & 32 & \\
\hline Ateus & 8 & 4 & \\
\hline Outros & & 4 & \\
\hline Estado civil & & & 0,714 \\
\hline Solteiras & 23 & 20 & \\
\hline União estável & 72 & 55 & \\
\hline Grau de instrução & & & 0,004 \\
\hline Fundamental incompleto & 45 & 18 & \\
\hline Fundamental completo & 21 & 30 & \\
\hline M édio & 29 & 26 & \\
\hline Superior completo & - & 1 & \\
\hline Doenças pessoais & & & 0,348 \\
\hline Sim & 23 & 23 & \\
\hline Não & 72 & 52 & \\
\hline Operações prévias & & & 0,892 \\
\hline Sim & 12 & 10 & \\
\hline Não & 83 & 65 & \\
\hline Alergias & & & 0,859 \\
\hline Sim & 10 & 10 & \\
\hline Não & 85 & 65 & \\
\hline Tabagismo & & & 0,604 \\
\hline Sim & 14 & 9 & \\
\hline Não & 81 & 66 & \\
\hline Etilismo & & & 0,415 \\
\hline Sim & 10 & 11 & \\
\hline Não & 85 & 64 & \\
\hline
\end{tabular}

atividade laborativa domiciliar. Também não houve diferença entre a via de parto ea atividade profissional das pacientes $(p=0,3126)(O R=$ 1,37; 0,71< OR <2,63). (Tabela 1).

Em relação ao grau de instrução materna, foi observada maior incidência de partos por via vaginal nas pacientes queestudaram atéo ensino fundamental incompleto $(p=0,0045)$.

Observou-se que $23(24,2 \%)$ pacientes do Grupo 1 e23 (30,6\%) pacientes do Grupo 2 apresentavam doenças, sem diferença entre os gru$\operatorname{pos}(p=0,348)(O R=0,72 ; 0,35<0 R<1,50)$. As moléstias mais frequentes em ambos os grupos foram asma, hipertensão arterial sistêmica, depressão, hipotireoidismo e gastrites. Duas pacientes em cada grupo foram tratadas de infertilidade. Uma paciente do Grupo 2 tivera leucemia linfocítica aguda aos cinco meses de idade, com cura completa.

Procedimentos cirúrgicos prévios ocorreram em doze ( $12,6 \%$ ) pacientes do Grupo 1 (cirurgia

plástica para tratamento de quelóide, drenagem de abscesso pulmonar, drenagem abscesso do braço, amigdalectomia, correção cirúrgica de fratura de fêmur, tireoidectomia, desobstrução de canais lacrimais, artrodese de coluna lombar, apendicectomia - dois casos, ressecção de endometrioma e colecistectomia) e em dez (13,3\%) pacientes do Grupo 2 (cirurgia plástica para lábio leporino, mamoplastia - dois casos, correção cirúrgica de fratura de fêmur, adenoidectomia, troca de válvula mitral, nodulectomia mamária, miomectomia uterina, gastroplastia redutora, hernioplastia umbilical) sem diferença entre si $(p=0,892)(O R=0,94 ; 0,35<O R<$ $2,53)$. H ouvemaior incidência deoperações esté ticas nas pacientes submetidas a parto cesáreo (três casos no Grupo 2 e nenhum no Grupo 1) $(p=0,041)$. Em relação ao uso de medicamentos, não houve diferença entre os dois grupos ( $p$ $=0,129$ ). Uma paciente do Grupo 1 relatou fazer uso de cocaína e crack e outra de maconha.

Alergia foi verificada em dez pacientes de cada grupo, não havendo diferença entre elas ( $p=$ 0,572 ). Tabagismo foi constatado em $14,7 \%$ das pacientes do Grupo 1 e $12 \%$ das do Grupo 2 ( $p=$ $0,604)(O R=1,27 ; 0,48<O R<3,41)$. Não houve diferença na incidência de etilismo entre os dois grupos (dez pacientes no Grupo 1 eonzeno Grupo 2) $(p=0,68)(0,25<0 R<1,87)$.

Em relação à história gestacional, 38 pacientes do Grupo 1 e 31 do Grupo 2 eram primiges$\operatorname{tas}(p=0,8604)(O R=0,95 ; 0,49<O R<1,84)$. $N$ ão houve diferença entre o número pregresso degestaçõese partos entreos grupos $(p=0,9840)$. $\mathrm{H}$ istória de abortamento foi verificada em catorze pacientes do Grupo 1 e dezesseis pacientes do Grupo 2 ( $p=0,2626)(O R=0,64 ; 0,27<O R<$ 1,51). 0 número de gestações variou de um a doze no Grupo 1 e de um a sete no Grupo 2.

H ouve incidência pregressa maior de partos vaginais no Grupo 1 e de cesáreas no Grupo 2 ( $p$ $<0,001)(O R=104,00 ; 21,11<0 R<610,99)$.

0 número de consultas pré natais variou de um a doze (média de6,559 $\pm 0,2381$ ) no Grupo 1 e de um a dezoito no Grupo 2 (média de 7,877 \pm $0,3047)$. Foi verificada maior número de consultas no Grupo 2 ( $p=0,0007)$.

A Tabela 2 mostra o tipo de parto desejado pelas gestantes. Observou a vontade de 78 (82,1\%) das mulheres do Grupo 1 de terem sido submetidas a partos vaginais contra 28 (37,3\%) das mulheres do Grupo 2 de terem sido submetidas a cesáreas $(p=0,0002)(O R=4 ; 1,77<O R$ $<9,17)$. Tanto as mulheres submetidas a partos vaginais quanto as submetidas a cesáreas justifi- 
caram a escolha do tipo de parto, relatando a recuperação mais rápida e o medo de sentir dor. Outras justificações menos frequentes foram preocupação com a estética, vontade de fazer salpingotripsia no mesmo ato operatório e por seguir orientação do obstetra. Observa-sequehouve uma maior incidência de indicação obstétrica para a escolha de parto por via cesárea $(p=$ 0,00009) (Tabela 3).

0 uso do fórceps foi necessário em sete $(7,4 \%)$ pacientes do Grupo 1 . H ouve maior incidência de apresentação fetal cefálica em ambos os grupos. Entretanto, o Grupo 2 apresentou um número maior de partos com apresentação pél-

Tabela 2. Dados relacionados às condições gestacionais das pacientes submetidas a partos por via vaginal (Grupo 1) e por cesárea (Grupo 2).

\begin{tabular}{lrrl}
\hline & Grupo 1 & Grupo 2 & Valor p \\
\hline Partos prévios & & & $<0,0001$ \\
$\quad$ Vaginal & 32 & 6 & \\
Cesárea & 36 & \\
$\quad$ Ambos & 2 & 2 & \\
Tipo de parto desejado & & & 0,0002 \\
$\quad$ Vaginal & 78 & 42 & \\
Cesárea & 13 & 28 & \\
Indiferente & 4 & 5 & \\
Tipo de apresentação & & & 0,0006 \\
$\quad$ Cefálica & 93 & 64 & \\
Pélvica & 1 & 11 & \\
$\quad$ Transversa & 1 & - & \multirow{2}{*}{0,0001} \\
Tipo de anestesia & & & \\
Raquidiana & 12 & 69 & \\
Peridural & 11 & 6 & \\
Local & 50 & - & \\
Nenhuma & 22 & - & \\
\end{tabular}

$\operatorname{vica}(p=0,0006)(O R=15,98 ; 2,05<0 R<339,33)$ (Tabela 2).

As indicações mais frequentes pela opção do parto por cesárea foram desproporção cefalopélvica (32 casos), cesárea prévia (dez casos), macrossomia fetal (oito casos), ausência de dilatação do colo do útero (sete casos), cesárea eletiva (sete casos), pré-eclâmpsia(seis casos), distócias decontração (três casos), hemorragias (dois casos).

A maior parte das pacientes do Grupo 1 foi submetida a anestesia local (cinquenta casos) ou nenhuma anestesia (22 pacientes). Já as do Grupo 2 foram submetidas à anestesia raquidiana (69 casos) $(p<0,0001)(0 R=0,09 ; 0,02<0 R<$ 0,35 ) (Tabela 2). H ouveesclarecimento do tipo de parto pelo obstetra em apenas dez pacientes do Grupo 1 e sete pacientes do Grupo $2(p=0,07)$.

$A$ idade gestacional variou entre 35 e 42 semanas no Grupo 1 (média de 38,95 $\pm 1,27$ ) e entre 35 e 41 semanas no Grupo 2 (média de $38,83 \pm 1,29)$, sem diferença entre si ( $p=0,549$ ).

A Tabela 4 mostra as doenças relacionadas à gestação e ao puerpério nas pacientes de ambos os grupos. Essas doenças foram verificadas em 66 pacientes do Grupo 1 e 59 pacientes do Grupo $2(p=0,17)(O R=0,62 ; 0,29<0 R<1,32)$.

Em relação às complicações maternas decorrentes do parto, foi verificada infecção cirúrgica em quatro pacientes (duas do Grupo 1 eduas do Grupo 2). Em duas pacientes do Grupo 1, houve laceração vaginal, que necessitou sutura. H ouve dor intensa no pós-operatório nas pacientes submetidas a parto cesárea (oito casos) quando comparadas com as pacientes do Grupo 1 (dois casos) $(p=0,018)(O R=0,18 ; 0,03<0 R<0,96)$.

A Tabela 5 mostra as malformações congênitas verificadas em seis pacientes do Grupo 1 enove pacientes do Grupo 2: distopias testiculares (cinco casos), hemangiomas (quatro casos), polidac-

Tabela 3. Justificativa para escolha do tipo de parto nas pacientes submetidas a partos por via vaginal (Grupo 1) e por cesárea (Grupo 2).

\begin{tabular}{|c|c|c|c|c|}
\hline & \multicolumn{2}{|c|}{ Grupo 1} & \multicolumn{2}{|c|}{ Grupo 2} \\
\hline & $\mathrm{N}$ & $\%$ & $\mathrm{~N}$ & $\%$ \\
\hline Recuperação mais rápida & 47 & 49,4 & 27 & 36,0 \\
\hline M enos medo/dor & 33 & 34,7 & 12 & 16,0 \\
\hline Preocupação estética & 1 & 1,0 & 2 & 2,7 \\
\hline Realização simultânea de salpingotripsia & 5 & 5,3 & 11 & 14,7 \\
\hline Indicação do obstetra * & 5 & 5,3 & 23 & 30,6 \\
\hline M edo de cirurgia & 4 & 4,3 & - & - \\
\hline
\end{tabular}

$* p=0,00009$ 
Tabela 4. D oenças mais frequentes relacionadas à gestação e ao puerpério nas pacientes submetidas a partos por via vaginal (Grupo 1 ) e por cesárea (Grupo 2).

\begin{tabular}{lrr}
\hline \multicolumn{1}{c}{ Doenças * } & Grupo 1 & Grupo 2 \\
\hline Anemia & 23 & 17 \\
Ameaça parto pré-termo & 7 & 4 \\
Hemorragias & 12 & 11 \\
Infecção do trato urinário & 34 & 19 \\
Hipertensão arterial sistêmica & 4 & 4 \\
Pré-eclâmpsia & 5 & 6 \\
Diabetes gestacional & 1 & 3 \\
Candidíase & 8 & 12
\end{tabular}

Obs: Algumas pacientes apresentaram mais de uma complicação durante o período gestacional e puerpério. *: $p=0,17$ (testet deStudent)

Tabela 5. M alformações congênitas observadas nos pacientes submetidos a parto por via vaginal (Grupo 1) e por cesárea (Grupo 2).

\begin{tabular}{lcc}
\hline & Grupo 1 & Grupo 2 \\
\hline Distopias testiculares & 2 & 3 \\
Hemagioma & 2 & 2 \\
Polidactilia & 1 & 2 \\
Doença cardíaca congênita & 1 & 1 \\
Síndrome de Cornélia de Lange & - & 1
\end{tabular}

tilia (três casos), doença cardíaca congênita (dois casos), síndrome de Cornélia de Lange(um caso). $N$ ão houve diferença entre a presença das malformações ea escolha do tipo de parto $(p-0,19)$.

0 peso ao nascer variou de $2.040 \mathrm{~g}$ a $4.420 \mathrm{~g}$ ( $3.146 \pm 439 \mathrm{~g}$ ) no Grupo 1 e de $1.915 \mathrm{~g}$ a $4.290 \mathrm{~g}$ ( $3.139 \pm 495 \mathrm{~g})$ no Grupo 2, não diferindo entre os grupos $(p=0,929)$. A estatura média do Grupo 1 foi de $48,6 \pm 1,9 \mathrm{~cm}$ ea do grupo 2 de $48,1 \pm$ $1,8 \mathrm{~cm}$, também sem diferença entre os grupos $(p=0,087)$.

O Apgar de $76,8 \%$ dos recém-nascidos do Grupo 1 e $76 \%$ dos do Grupo 2 no primeiro minuto foi igual ou superior a $8(p=0,89)$ e $82,1 \%$ dos recém-nascidos do Grupo 1 e 81,3\% dos do Grupo 2 apresentaram Apgar após cinco minutos igual ou superior a $9(p=0,88)$.

0 tempo entre a primeira contração uterina, anterior ao início do trabalho de parto, variou de trinta minutos a 48 horas no Grupo 1 com mediana de seis horas. Já no Grupo 2, 53,3\% (40 mulheres) tiveram indicação e foram submetidas a cesáreas antes de estarem em trabalho de parto. Circular de cordão ocorreu em oito recém-nascidos do Grupo 1 eem cinco do Grupo 2 $(p=0,66)$.

Traumas obstétricos foram verificados em catorze recém-nascidos do Grupo 1 e em sete do Grupo 2 ( $p=0,28$ ), com escoriações e equimoses. No Grupo 1, além das escoriações e equimoses em oito casos, houve cinco fratura declavícula e uma lesão do plexo braquial. Constatou-se hiperbilirrubinemia neonatal em quatro pacientes do Grupo 1 e sete pacientes do Grupo 2, todos com excelente resposta à fototerapia ( $p=$ $0,17)$. Insuficiência respiratória ocorreu em seis recém-nascidos de cada grupo $(p=0,67)$. Desses, um paciente do Grupo 1 e dois do Grupo 2 foram intubados e encaminhados ao CTI ( $p=$ $0,63)$. Dificuldade de sucção foi encontrada em quatro pacientes de cada grupo $(p=0,73)$.

0 tempo de internação hospitalar da gestante variou de dois a quatro dias no Grupo 1 e de dois a oito dias no Grupo 2. A média de internação dos recém-nascidos foi maior no Grupo 2 (3,43 $\pm 0,70$ dias) quando comparados com os recém-nascidos de parto por via vaginal $(2,71 \pm$ 0,67 dias) $(p<0,0001)$.

\section{Discussão}

Este estudo analisou prospectivamente a morbidade neonatal e materna de acordo com o tipo departo. Resultados comparados entre parto por via vaginal e parto cesáreo são inerentemente parciais, já que a parturiente e o recém-nascido submetidos a um desses dois métodos têm fatores de risco diferentes.

Segundo Kilsztajn et al. ${ }^{6}$, a taxa de cesarianas é maior entre as mulheres com maior grau de instrução. Em nosso estudo, constatou-se que $76 \%$ das mulheres submetidas a parto cesariana tinham tempo de estudo iguais ou superior a nove anos, enquanto apenas $52 \%$ das mulheres que realizaram parto por via vaginal tinham esse grau de instrução.

Ao contrário do que foi verificado por Villar et al. ${ }^{5}$, em nosso estudo, primigestação não foi associada a uma taxa maior de entrega cesárea, sendo a proporção de primigestas no Grupo 2 semelhante ao Grupo 1. A incidência pregressa maior de partos vaginais em mulheres multíparas no Grupo 1 e de cesáreas no Grupo 2 sugere 
que o tipo de primeiro parto é um fator de importância para definir a via de parto seguinte.

Sabe-se que as taxas de parto cesariano no Brasil são elevadas, alcançando $32 \%$ no setor público, segundo estudo realizado por Kilsztajn et al. ${ }^{6}$, sem que isso reflita a preferência das muIheres por esse tipo de parto. Em nosso estudo, cerca de $56 \%$ das mulheres submetidas a cesariana desejavam realizar parto por via vaginal. Pelo fato de a pergunta ter sido feita após o parto, pode ter havido alguma influência em relação à satisfação com o tipo de parto que essas mulheres tiveram ${ }^{6}$. Por outro lado, nosso estudo está em concordância com o estudo realizado por Potter et al. ${ }^{10}$, queindicaram ser o desejo por parto cesariano menor do que se esperava. Portanto, 0 aumento nas taxas de cesarianas não pode ser justificado pelo aumento na demanda pelo parto cirúrgico. 0 desejo da gestante de realizar seu parto com o obstetra que a acompanhou durante o pré-natal pode ser um dos motivos de muitas mães desejarem parto cesárea, já que muitos dos médicos da rede hospitalar pública trabaIham em regime deplantão epoderia não coincidir o trabal ho de parto com o dia em que o obstetra está na maternidade. De acordo com o presente trabalho, apenas dez pacientes do Grupo 1 e sete do Grupo 2 foram esclarecidas adequadamente sobre o tipo de parto. Essa falta de informação pode levar em grande parte dos casos ao medo e suas consequências.

A maioria das mulheres que desejava real izar parto cesariano justificou a escolha pelo medo de sentir dor. De fato, 52,6\% das pacientes submetidas a parto por via vaginal tiveram apenas anestesia local e 23,1\% não foram submetidas a qualquer tipo de anestesia. Por outro lado, esse medo de sentir dor também foi a justificativa de algumas pacientes submetidas aos partos por via cesárea. Por trás desse sentimento, há falta de esclarecimento por parte do médico sobre os tipos de parto ea possi bilidade de anestesia e analgesia em ambos os casos, que previne esse desconforto.

Em concordância com resultados encontrados por Villar et al. ${ }^{5}$, as justificações mais frequentes para o parto cesáreo foram desproporção cefalopélvica, cesárea prévia edistócia decontração. M olkenboer et al. ${ }^{14}$ observaram que partos cesáreos são preferíveis para fetos com apresentação pélvica.

Foi verificado neste estudo que, mesmo com uma média de consultas pré-natais de 6,5 no Grupo 1 e 7,87 no Grupo 2, a maioria das gestantes não foi esclarecida sobre os tipos de parto.
O papel do obstetra não é apenas prevenir e curar doenças relacionadas à gestação, mas também esclarecer as dúvidas das pacientes e explicar a conveniência de suas decisões, que devem ser discutidas com as pacientes.

Ao contrário do que poderia esperar-se, não houve diferenças entre a média de idade gestacional (IG) entre os tipos de parto. A explicação para tal expectativa está no fato de muitos médicos agendarem a cesariana segundo sua conveniência. Uma das hipóteses deste estudo era 0 possível comprometimento fetal provocado pela diminuição do tempo de gestação no parto por cesárea. No entanto, essa pressuposição não foi confirmada, pois o Apgar no primeiro minuto foi igual ou maior que 8 na maioria dos recémnatos, sem diferença entre os dois grupos.

As complicações maternas decorrentes do parto também não diferiram entre os dois tipos de parto à exceção da dor, que foi mais intensa nas pacientes submetidas a cesarianas. Segundo Rortveit et al. ${ }^{15}$, a taxa de incontinência urinária pósparto é maior entre pacientes com parto por via vaginal. Nesteestudo, não foi possível avaliar morbidades como incontinência urinária e fecal porque elas não foram registradas nos prontuários das pacientes e não são queixas registradas durante as primeiras consultas ginecológicas pós-parto.

$\mathrm{Na}$ hipótese original deste estudo, esperavase encontrar o índice de Apgar menor no Grupo 2 do que no Grupo 1,tendo em vista a relativa prematuridadedos neonatos por cesárea. Segundo Villar et al. ${ }^{5}$, há relação direta entre a incidência de síndrome da angústia respiratória do recém-nascido e parto cesariana. No entanto, no presenteestudo não houve diferença entreosgrupos para os índices de Apgar no primeiro equinto minutos nascimento, indicando o bom estado de saúde do recém-nato.

Por outro lado, houve associação entre parto por via vaginal e morbidade neonatal. Segundo Giglio et al. ${ }^{9}$ a associação entre parto normal e aumento da morbimortalidade neonatal decorreria de viés de seleção devido à distribuição na rede hospitalar pública de pacientes com maior risco gestacional. Para evitar tendências de seleção, foram excluídas parturientes com complicações gestacionais e com nascimento pré-termo. Semelhantementeao encontrado por M cFarland et al. ${ }^{13}$, houve efeito protetor do parto cesariano para o neonato já quea proporção de traumas obstétricos mais graves foram encontrados no Grupo 1. No estudo de alguns dados, dada a possibilidade de diferentes variáveis, houveum $n$ pequeno, dificultando a avaliação estatística. 
O tempo de internação hospitalar do recémnascido no Grupo 2 foi maior do que no Grupo 1. Isso porqueparturientes pós-cesárea precisam de maior tempo de observação pós-cirúrgica e com isso a maternidade prolonga a internação do recém-nato para manter a amamentação. Em relação à amamentação, seria interessante estudar os lactentes por um período entre seis meses a um ano, uma vez que eventuais problemas poderiam surgir em idades maiores que as estudadas, embora decorrentes muitas vezes de múltiplos fatores - amamentação materna exclusiva, peso, estatura, patologias, desenvolvimento, alimentação, etc.

\section{Conclusão}

Pode-se concluir, a partir dos resultados obtidos neste estudo, que existe maior morbidade neonatal em recém-natos de parto por via vaginal quando comparada com neonatos de cesárea. No entanto, não houve diferença entre os grupos ao se tratar de afecções respiratórias e mortalidade neonatal. Já a morbidade materna é maior em puérperas com parto cesariana.

\section{Colaboradores}

PO Cardoso trabalhou na concepção teórica, elaboração, organização e execução das oficinas, revisão bibliográfica e redação final do texto. LR Alberti participou da elaboração, revisão bibligráfica e redação final do texto. A Petroianu trabalhou na concepção teórica, elaboração, organização das oficinas, revisão bibliográfica eredação final do texto. 


\section{Referências}

1. M artins-Costa S, Ramos JGL. A questão das cesarianas. Rev Bras Ginecol Obstet 2005; 27: 571-574.

2. Jimenez V. La Césarienne sur demande une manifestation de la culture de la peur? Le M édecin du Québec 2005; 40:65-69.

3. Liu S, Heaman M, Joseph KS, Liston RM, Huang L, Sauve R, Kramer MS. Risk of M aternal Postpartum Readmission Associated With Mode of Delivery. Am J Obstet Gynecol 2005; 105:836-842.

4. Belizán JM, Althabe F, Barros FC, Alexander S. Rates and implications of caesarean section in Latin American. B M ed J 1999; 319:1397-1402.

5. Villar J, Valladares E, Wojdyla D, Zavaleta N, Carroli G, Velazco A, Shah A, Compodónico L, Bataglia $V$, Faundes $A$, Langer $A$, Narváez $A$, D onner $A$, Romero M, Reynoso S, de Pádua KS, Giordano $D$, Kublickas M, Acosta A, WHO 2005 global survey on maternal and perinatal health research group. Caesarean delivery rates and pregnancy outcomes: the 2005 WHO global survey on maternal and perinatal health in Latin America. Lancet 2006; 367:1819-1940.

6. Kilsztajn S, Carmo M, Machado Jr L, Lopes E, Lima L. Caesarean sections and maternal mortality in São Paulo. Eur J Obstet Gynecol Reprod Biol 2007; 132:64-69.

7. Shearer E. Cesarean section: medical benefits and cost. Soc Sci M ed 1993; 37:1223-1231.

8. Béhague DP, Victora CG. Consumer demand for cesarean section in Brazil. B M ed J 2002; 324:942-948.

9. Giglio MRP, Lamounier JA, Neto OLM. Via de parto e risco para mortalidade neonatal em Goiânia no ano 2000. Rev. Saude Publica 2005; 39:350-357.

10. Portter JE, Berquó E, Leal F, Hopkins K, Formiga CC. Unwanted caesarean section among public and private patients in Brazil. B M ed J 2001; 323:11551158.

11. Quadros LGA. Brazilian obstetricians are pressured to perform caesarean section. B M ed J 2000; 320:1073-1073.

12. Murray SF. Relation between private health insurance and high rate of caesarean section in Chile. $\mathrm{B}$ M ed J 2000; 321:1501-1505.

13. McFarland LV, Raskin M, Daling JR, Benedetti TJ Erb/Duchenne's palsy: a consequence of fetal macrosomia and method of delivery. Obstetrics \& Gynecology 1986; 68:784-788.
14. Molkenboer JF, Reijners EP, Nijhuis JG, Roumen FJ. M oderate neonatal morbidity after vaginal term breech delivery. J Matern Fetal Neonatal Med 2004; 16:357-361.

15. Rortveit G, Daltveit AK, H annestad YS, Hunskaar S. Urinary Incontinence after Vaginal Delivery or $\mathrm{Ce}$ sarean Section. N Engl J M ed 2003; 348:900-907.

16. Rouse DJ, Owen J. Prophylactic cesarean delivery for fetal macrosomia diagnosed by means of ultrasonography - A Faustian bargain? Am J Obstet Gynecol 199; 181:332-338.

17. Zanardo V, Simbi AK, Franzoi M. Neonatal respiratory morbidity risk and mode of delivery at term. Acta Paediatr 2004; 93:643-647.

18. Nicoll AE, Black C, Powls $A$, Mackenzie F. An audit of neonatal respiratory morbidity following elective caesarean section at term. Scott M ed J 2004; 49:22-25.

19. Weiderpass E, Barros FC, Victora CG, Tomasi E, Halpern R. Incidência e duração da amamentação conforme o tipo de parto: estudo longitudinal no Sul do Brasil. Rev. Saude Publica 1998; 32:225-231.

20. Burrows LJ, Meyn LA, Weber AM . Maternal Morbidity Associated With Vaginal Versus Cesarean Delivery. Am J Obstet Gynecol 2004; 103:907-912.

21. M artins-Costa, SH, Ramos JGL, Serrano YLG. Trauma during pregnancy. Rev Bras Ginecol 2005; 27:505508.

22. Golfier F, Vaudoyer F, Ecochard R, Champion F, Audra P, Raudrant D. Planned vaginal delivery versus elective caesarean section in singleton term breech presentation: a study of 1116 cases. Eur J Obstet Gynecol Reprod Biol 2001; 98:186-192.

23. Petroianu A. Pesquisa em medicina. In: Petroianu A, organizador. Ética, moral e deontologia médicas. Rio de Janeiro: Guanabara Koogan; 2000. p. 174-178.

Artigo apresentado em 21/10/2007

Aprovado em 30/06/2008

Versão final apresentada em 04/07/2008 